\title{
Dynamics of pick-up ions in collisionless velocity shears
}

\author{
E. Pérez-Tijerina ${ }^{1}$, M. Reyes-Ruiz ${ }^{2}$, H. Pérez-de-Tejada ${ }^{3}$, and H. Durand-Manterola ${ }^{4}$ \\ 1 Posgrado en Fisica Espacial, Instituto de Geofisica, UNAM Km. 103 Carr. Tijuana-Ensenada, 22860 Ensenada, \\ B.C. México \\ 2 Instituto de Astronomía, UNAM, Km. 103 Carr. Tijuana-Ensenada, 22860 Ensenada, B.C. México \\ 3 Instituto de Geofísica, UNAM, Ensenada, B.C. México \\ 4 Instituto de Geofísica, UNAM, México D.F., México
}

Received 20 March 2001 / Accepted 15 January 2002

\begin{abstract}
We study the motion of charged particles in large-scale velocity shears that are produced in the interaction of magnetized plasma winds and plasma obstacles. The purpose of the analysis is to account for the observation of strongly energetic contaminant ions in the region of interaction of the solar wind with planetary/cometary non-magnetic ionospheric obstacles (Venus, Mars, comets). The convective electric field set up by the streaming plasma is incorporated to the equation of motion of ions born in a velocity shear. Neglecting collisions and the back reaction of the contaminant particles on the wind, the trajectories of the particles are computed as a function of the shear properties as well as of the mass of the ions and the magnetic field configuration. For a linear dependence of the wind velocity across the shear, the problem is solved analytically and we find that the particle velocity can have either a purely oscillatory behavior or grow exponentially with time depending on the value of a dimensionless parameter proportional to the product of the velocity gradient and the cyclotron frequency of the ion trajectories. In the latter case a strong acceleration of the contaminant ions can be achieved. Adopting magnetic field and flow properties appropriate for cometary and planetary environments, we explore the potential importance of the mechanism discussed to explain the presence of superthermal ions and filamentary structures in such regions.
\end{abstract}

Key words. acceleration of particles - plasmas - comets - planets and satelites

\section{Introduction}

It has been suggested that the tenuous solar wind that flows around non-magnetic ionospheric obstacles gives rise to boundary layers over their magnetic polar regions (these are defined by the orientation of the interplanetary magnetic field), similar to those resulting from viscous flows that stream around spherical objects (Pérez-de-Tejada 1986). Evidence for this view has been provided by multiple satellite observations in Venus, Mars and several comets. However, the collisionless nature of the solar wind has led to issues related to the physical sources of the anomalous viscosity that are responsible for momentum transport across the boundary layer. Today, the observational evidence that is available has led to suggestions on microscopic processes (e.g. wave-particle interactions) that could account for momentum transfer in the collisionless solar wind (Shapiro et al. 1995). As a result, it is important to carry out a thorough investigation of the likely consequences of the existence of boundary

Send offprint requests to: M. Reyes-Ruiz, e-mail: maurey@astrosen.unam.mx layers and verify the consistency of momentum transfer with regard to other observational data.

In the present study we will analyze the motion of individual ions born in the vicinity of ionospheric obstacles (mass loading) subject to the convective electric field set up by the solar wind and in the presence of a velocity shear. This problem was initially examined by Parker (1958) for the case in which the wind velocity, the magnetic field, and the velocity gradient are transverse to each other. The pick-up ions execute cycloidal motion at the local velocity of the flow within the boundary layer. A later study was conducted by Pérez-de-Tejada \& Durand-Manterola (1996, hereafter referred to as PD96) who revised this problem and calculated the ion density expected from the particle trajectories. From mass flux conservation these authors derived a gradient for the pickup ion density that is opposed to the velocity gradient and also derived values that are consistent with density measurements made in the vicinity of comet GiacobinniZinner (Bame et al. 1986). Our main interest will now be to extend the analysis of ion trajectories to arbitrary orientations between the magnetic field and the velocity 


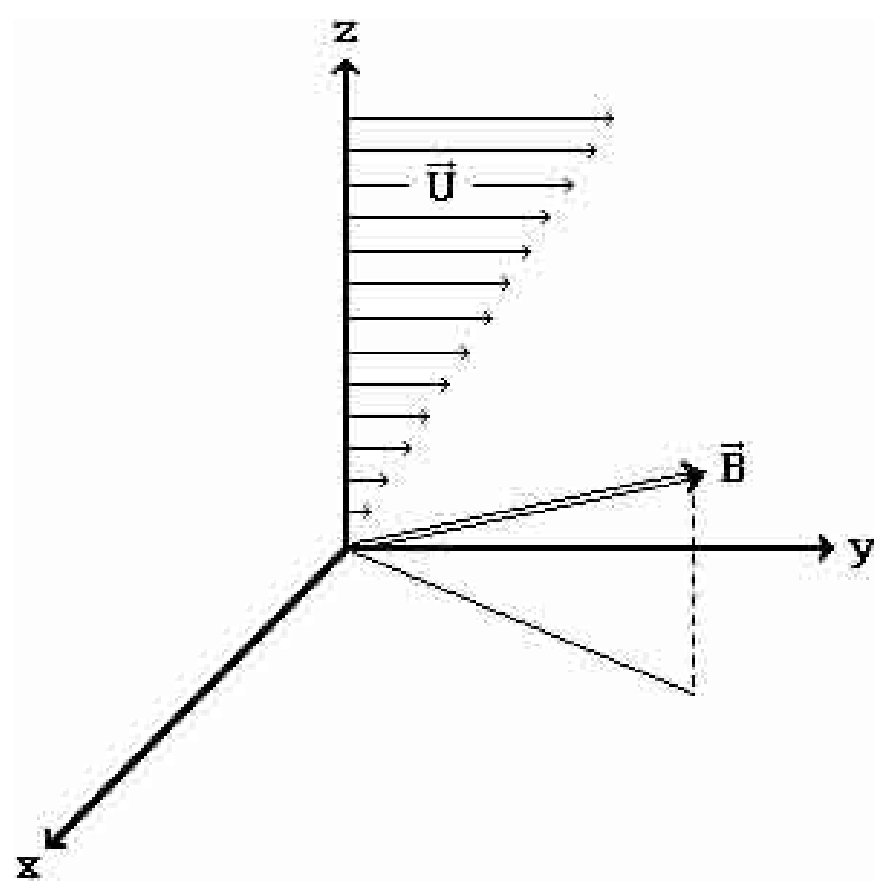

Fig. 1. Basic geometry of the problem being solved. The magnetic field, $\boldsymbol{B}$, has an arbitrary orientation with the respect to the flow $\boldsymbol{U}$ which varies only in the $z$-direction.

gradient and derive an analytical solution for the constant velocity gradient case as well as numerical solutions suitable for classical boundary layers. While a linear profile for the flow velocity is supported by observations in comets (PD96) it is important to study different magnetic field configurations as well as to examine the sensitivity of the results to other forms of the velocity gradient using theoretically justified profiles. In light of our results we will discuss novel potential applications of these ideas to explain the acceleration of charged particles in space and astrophysical phenomena.

The paper is organized as follows: Sect. 2 we present the formulation of the problem to be solved. Section 3 deals with the analytic solution of the equation of motion for charged particles in linear velocity shears. In Sect. 4 we discuss the results obtained focusing on the process of particle acceleration. In Sect. 5 we show that our results may have potential applications in the study of the interaction of the solar wind with unmagnetized bodies, leaving a detailed, quantitative analysis for future contributions. And finally, in Sect. 6 we summarize our results and conclusions.

\section{Formulation of the problem}

We consider the case in which a uniform magnetic field, frozen to a streaming plasma, is oriented arbitrarily with respect to a one-dimensional velocity gradient along a height coordinate over an immobile obstacle. The basic configuration, with a linear velocity profile, is illustrated in Fig. 1.
Using Cartesian coordinates the flow streams along the $y$ axis and the velocity gradient is directed in the $z$ axis. The streaming plasma flows with velocity $\boldsymbol{U}=[0, U(z), 0]$ carrying a "frozen-in" magnetic field $\boldsymbol{B}=\left[B_{x}, B_{y}, B_{z}\right]$. Our reference frame is considered at rest in the obstacle so that a convective electric field $\boldsymbol{E}=-(\boldsymbol{U} \times \boldsymbol{B}) / c$, is felt there. The equation of motion for an individual particle of mass $m$, charge $q$ and position $\boldsymbol{x}$, is:

$m \frac{\mathrm{d}^{2} \boldsymbol{x}}{\mathrm{d} t^{2}}=q \boldsymbol{E}+\frac{q}{c} \frac{\mathrm{d} \boldsymbol{x}}{\mathrm{d} t} \times \boldsymbol{B}$

whose components (with $\boldsymbol{x}=[x, y, z]$ ) are:

$\frac{\mathrm{d}^{2} x}{\mathrm{~d} t^{2}}=-\Omega_{z} U(z)+\Omega_{z} \frac{\mathrm{d} y}{\mathrm{~d} t}-\Omega_{y} \frac{\mathrm{d} z}{\mathrm{~d} t}$

$\frac{\mathrm{d}^{2} y}{\mathrm{~d} t^{2}}=\Omega_{x} \frac{\mathrm{d} z}{\mathrm{~d} t}-\Omega_{z} \frac{\mathrm{d} x}{\mathrm{~d} t}$

$\frac{\mathrm{d}^{2} z}{\mathrm{~d} t^{2}}=\Omega_{x} U(z)+\Omega_{y} \frac{\mathrm{d} x}{\mathrm{~d} t}-\Omega_{x} \frac{\mathrm{d} y}{\mathrm{~d} t}$

where,

$\Omega_{x}=\frac{q B_{x}}{m c}, \quad \Omega_{y}=\frac{q B_{y}}{m c}$ and $\quad \Omega_{z}=\frac{q B_{z}}{m c}$

and

$\Omega=\sqrt{\Omega_{x}^{2}+\Omega_{y}^{2}+\Omega_{z}^{2}}$.

While $\Omega$ is always positive $\Omega_{x}, \Omega_{y}$ and $\Omega_{z}$ can be negative depending on the sign of the charge and the respective field component.

We present results for the case in which the velocity depends linearly with distance above the obstacle and derive particle trajectories using an analytical solution. In the discussion section we will later analyze the problem with a velocity gradient as that resulting from a standard boundary layer in which the velocity increases as $z$ away from the obstacle.

The set of equations can be easily solved for a linear velocity gradient $U(z)=K z$ by taking their Laplace transform for a given set of initial conditions. In the following, we will assume that the particles start their motion from rest, at some point $\left[0,0, z_{0}\right]$ on the $z$-axis, i.e.

$x(t=0)=y(t=0)=0$

$\dot{x}(t=0)=\dot{y}(t=0)=\dot{z}(t=0)=0$.

These initial conditions are based on the physical problems we intend to study since the pick-up ions arise from a relatively small region, of the order of the size of the obstacle, in comparison with the scale of the flow. This context also justifies our assumption that ions are born at rest with respect to the obstacle since typical thermal velocities in planetary/cometary atmospheres are much smaller than the solar wind velocity. 


\section{Results}

The complete calculation of the solution of Eqs. (2)-(4) is straightforward but tedious. An extensive discussion of the method employed can be found in Pérez-Tijerina (1999) and leads to:

$x(t)=X_{1}\left(\mathrm{e}^{r_{1} t}-1\right)+X_{2}\left(\mathrm{e}^{r_{2} t}-1\right)+X_{3}\left(\mathrm{e}^{r_{3} t}-1\right)$

$y(t)=Y_{1}\left(\mathrm{e}^{r_{1} t}-1\right)+Y_{2}\left(\mathrm{e}^{r_{2} t}-1\right)+Y_{3}\left(\mathrm{e}^{r_{3} t}-1\right)$

$z(t)=Z_{1} \mathrm{e}^{r_{1} t}+Z_{2} \mathrm{e}^{r_{2} t}+Z_{3} \mathrm{e}^{r_{3} t}$

where $r_{1}, r_{2}, r_{3}$ are the roots of equation

$r^{3}+\left(\Omega^{2}-K \Omega_{x}\right) r+K \Omega_{y} \Omega_{z}=0$

and the coefficients $X_{i}, Y_{i}$ and $Z_{i}$ for $i=1,2,3$ are given by

$X_{i}=\left[-\frac{K\left(\Omega_{x} \Omega_{y}+r_{i} \Omega_{z}\right) z_{0}}{r_{i}\left(3 r_{i}^{2}+\Omega^{2}-K \Omega_{x}\right)}\right]$

$Y_{i}=\left[\frac{K\left(\Omega_{x}^{2}+\Omega_{z}^{2}\right) z_{0}}{r_{i}\left(3 r_{i}^{2}+\Omega^{2}-K \Omega_{x}\right)}\right]$

$Z_{i}=\left[\frac{\left(r_{i}^{2}+\Omega^{2}\right) z_{0}}{3 r_{i}^{2}+\Omega^{2}-K \Omega_{x}}\right]$.

The roots of the characteristic Eq. (10) are:

$r_{1}=-\frac{1}{2}\left(s_{1}+s_{2}\right)+i \frac{\sqrt{3}}{2}\left(s_{1}-s_{2}\right)$,

$r_{2}=-\frac{1}{2}\left(s_{1}+s_{2}\right)-i \frac{\sqrt{3}}{2}\left(s_{1}-s_{2}\right)$,

and

$r_{3}=s_{1}+s_{2}$,

where:

$s_{1}=\left[-\frac{K \Omega_{y} \Omega_{z}}{2}+\sqrt{\frac{\omega^{6}}{27}+\frac{\left(K \Omega_{y} \Omega_{z}\right)^{2}}{4}}\right]^{\frac{1}{3}}$,

$s_{2}=\left[-\frac{K \Omega_{y} \Omega_{z}}{2}-\sqrt{\frac{\omega^{6}}{27}+\frac{\left(K \Omega_{y} \Omega_{z}\right)^{2}}{4}}\right]^{\frac{1}{3}}$,

and,

$\omega^{2}=\Omega^{2}\left(1-\frac{K \Omega_{x}}{\Omega^{2}}\right)$.

According to the theory of cubic equations $r_{3}$ will be real, and $r_{1}$ will be the conjugate of $r_{2}$, if:

$\mathcal{D}=\left[\frac{\Omega^{2}}{27}\left(1-\frac{K \Omega_{x}}{\Omega^{2}}\right)\right]^{3}+\left(\frac{1}{2} K \Omega_{y} \Omega_{z}\right)^{2}>0$.

Under different conditions the three roots can be real or imaginary with equal or unequal values depending on whether the value of the discriminant is smaller, greater or equal to zero.
Hence, allthough the solutions (7)-(9) looks rather complicated, the possible values of $r_{1}, r_{2}$ and $r_{3}$ clearly indicate the existence of three qualitatively different types of solutions; purely oscillatory, purely exponential (growing or decaying) or a mixture of both. As discussed in PérezTijerina et al. (2000) a constant velocity drift can exist in oscillatory solutions which will be considered below.

The emergence of either type of solution depends on the velocity gradient $K$, the magnetic field components through $\Omega_{x}, \Omega_{y}$ and $\Omega_{z}$ and the particle properties, namely its mass and electrical charge. These characteristics of the flow and particle determine which of the forces present in Eqs. (2)-(4) dominates. For example, the particle will execute mainly gyromotion if the terms involving first order time derivatives of position, on the right hand side of Eqs. (2)-(4), greatly dominate over the terms leading to exponential acceleration (from the height dependent electric force) i.e. those involving $U(z)$. As a critical ratio between these two is overcome, or if the electric force has no component along the magnetic field, qualitatively different solutions are obtained.

We thus divide the presentation of our results into two main cases, $\mathcal{D} \geq 0$ and $\mathcal{D}<0$.

\subsection{Results for $\mathcal{D} \geq 0$}

A positive discriminant $(\mathcal{D}>0)$ implies that $s_{1}$ and $s_{2}$ are real. In this case the solution can be written as:

$x(t)=\left(2 A_{x} \cos b t-2 B_{x} \sin b t+C_{x} \mathrm{e}^{-3 a t}\right) \mathrm{e}^{a t}-2 A_{x}-C_{x}(21)$

$y(t)=\left(2 A_{y} \cos b t-2 B_{y} \sin b t+C_{y} \mathrm{e}^{-3 a t}\right) \mathrm{e}^{a t}-2 A_{y}-C_{y}(22)$

$z(t)=\left(2 A_{z} \cos b t-2 B_{z} \sin b t+C_{z} \mathrm{e}^{-3 a t}\right) \mathrm{e}^{a t}$

where,

$A_{x}=\frac{X_{1}+X_{2}}{2}$,

$B_{x}=\frac{X_{1}-X_{2}}{2 i}$,

and,

$C_{x}=X_{3}$,

with similar expressions for $A_{y}, B_{y}, C_{y}$ and $A_{z}, B_{z}, C_{z}$. The "frequencies" $a$ and $b$ result from writting the roots of Eq. (10) as:

$r_{1}=a+i b$

$r_{2}=a-i b$

and

$r_{3}=-2 a$

Different solutions are obtained for the following cases: when $a=0$ and $b \neq 0$ we have purely oscillatory solutions. When $b=0$ and $a \neq 0$ they are purely exponential. And solutions consisting of growing or damped oscillations correspond to non-zero values for $a$ and $b$. We will analyze particular solutions for each of these cases separately. 


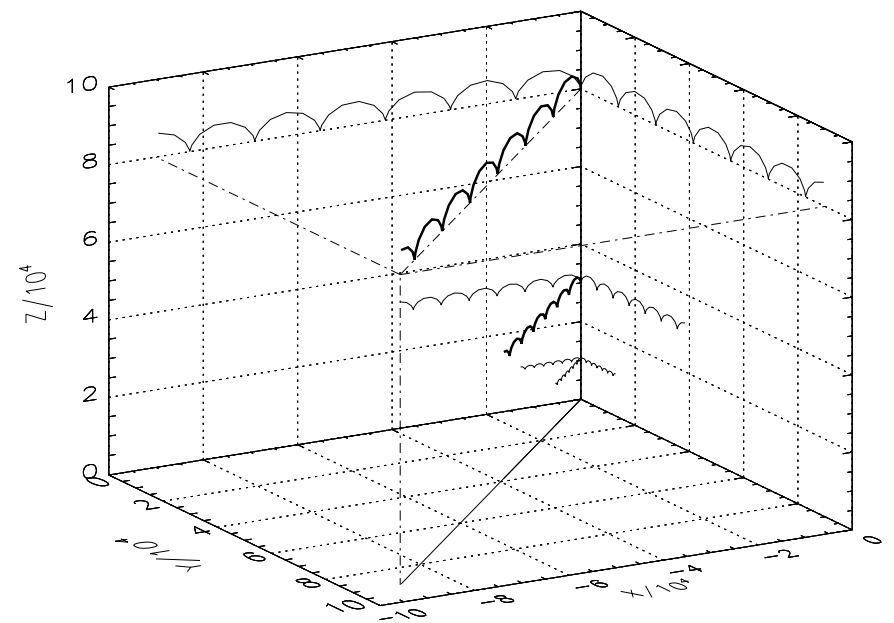

Fig. 2. Trajectories of oscillatory type for particles originating at 3 different initial positions for the case in which $\mathcal{D}>0$ with $\Omega_{x}=\Omega_{y}$ and $\Omega_{z}=0$.

\subsubsection{Oscillatory solutions}

The growth rate $a$ in Eqs. (21)-(23) will vanish when $s 1=$ $-s 2$. This can only be achieved if

$K \Omega_{y} \Omega_{z}=0$.

For a wind flow with velocity gradient this condition corresponds to a magnetic configuration in which the field is contained in the $x z$ or $x y$ planes. In either case, as well as when the field is along the $x$ axis, the solution will be purely oscillatory.

From Eqs. (14)-(16) and (27), (29) the frequency of the oscillatory motions is: $b=\omega$.

The coefficients in the solution (21)-(23) are easily obtained once a field configuration is specified. The calculation of the terms corresponding to the third root are obtained as a limit when $a \rightarrow 0$.

Particle trajectories have been derived and analyzed for magnetic configurations with $\boldsymbol{B}=\left(B_{x}, 0,0\right)$ and $\boldsymbol{B}=\left(B_{x}, B_{y}, 0\right)$ by Pérez-de-Tejada \& Durand-Manterola (1996) and Pérez-Tijerina et al. (2000) respectively. Our analysis for a general magnetic field orientation allows us to easily derive those results. Solutions in which the particles move on cycloidal trajectories drift in a direction parallel to $\boldsymbol{B} \times \nabla U$ are exemplified in Fig. 2. We refer the reader to the work of Pérez-Tijerina et al. (2000), and references therein for a careful analysis of these solutions. Here we focus, instead, on the novel results of our analysis.

It is worth mentioning that in the work of PérezTijerina et al. (1999) a parameter $\epsilon=K \Omega_{x} / \Omega^{2}$ is used to characterize the solutions in a manner analogous to the discriminant $\mathcal{D}$. When $K \Omega_{y} \Omega_{z}=0$ as is the case for the configurations studied by Pérez-Tijerina et al. (2000), the condition $\epsilon<1$ is equal to our inequality (20). The condition for $\epsilon$, equivalent to $\mathcal{D}>0$, in the case of general orientation of the magnetic field is

$\epsilon<1+27\left[\frac{K \Omega_{y} \Omega_{z}}{2 \Omega^{3}}\right]^{2 / 3}$

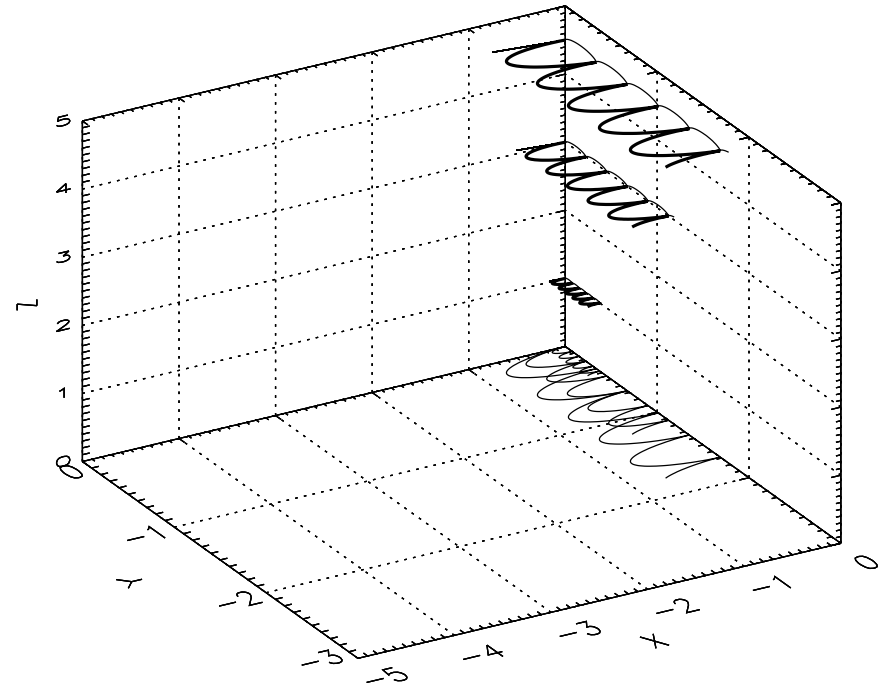

Fig. 3. Trajectories of oscillatory type for particles originating at 3 different initial positions for the case in which $\mathcal{D}>0$ with $\Omega_{x}=\Omega_{z}$ and $\Omega_{y}=0$.

which reduces to $\epsilon<1$ in the configurations previously studied.

Similar cycloidal trajectories are found in the case of magnetic field contained in the $x z$ plane. Solutions (21)(23) in this case simplify to:

$x(t)=\frac{K \Omega_{z} z_{\mathrm{O}}}{\omega^{2}}(\cos \omega t-1)$,

$y(t)=\frac{K \Omega^{2} z_{\mathrm{O}}}{\omega^{2}} t-\frac{K \Omega^{2} z_{\mathrm{O}}}{\omega^{3}} \sin \omega t$

and

$z(t)=\frac{\Omega^{2} z_{\mathrm{O}}}{\omega^{2}}-\frac{K \Omega_{x} z_{\mathrm{o}}}{\omega^{2}} \cos \omega t$.

Figure 3 shows a typical trajectory for a magnetic field $\boldsymbol{B}=\left(B_{x}, 0, B_{z}\right)$ and the additional restriction $\mathcal{D}>0$ or $\epsilon<1$. The exact value of $\epsilon$ is 0.05 .

\subsubsection{Exponential solutions}

Solutions purely exponential arise only when $\mathcal{D}=0$. In this case $s_{1}=s_{2}, b=0$ and $a$ is real. The parameter $\epsilon$ must be:

$\epsilon=\frac{K \Omega_{x}}{\Omega^{2}}=1+27\left[\frac{K \Omega_{y} \Omega_{z}}{2 \Omega^{3}}\right]^{2 / 3}$,

which is equal to one only when $K \Omega_{y} \Omega_{z}=0$. In such case however, both $a$ and $b$ are zero and one has a trivial solution.

When all field components are non-zero, satisfying Eq. (35), solutions as those shown in Fig. 4 are obtained. The form of such solutions is in general:

$x(t)=2 A_{x}\left(\mathrm{e}^{a t}-1\right)+C_{x}\left(\mathrm{e}^{-2 a t}-1\right)$,

$y(t)=2 A_{y}\left(\mathrm{e}^{a t}-1\right)+C_{y}\left(\mathrm{e}^{-2 a t}-1\right)$, 


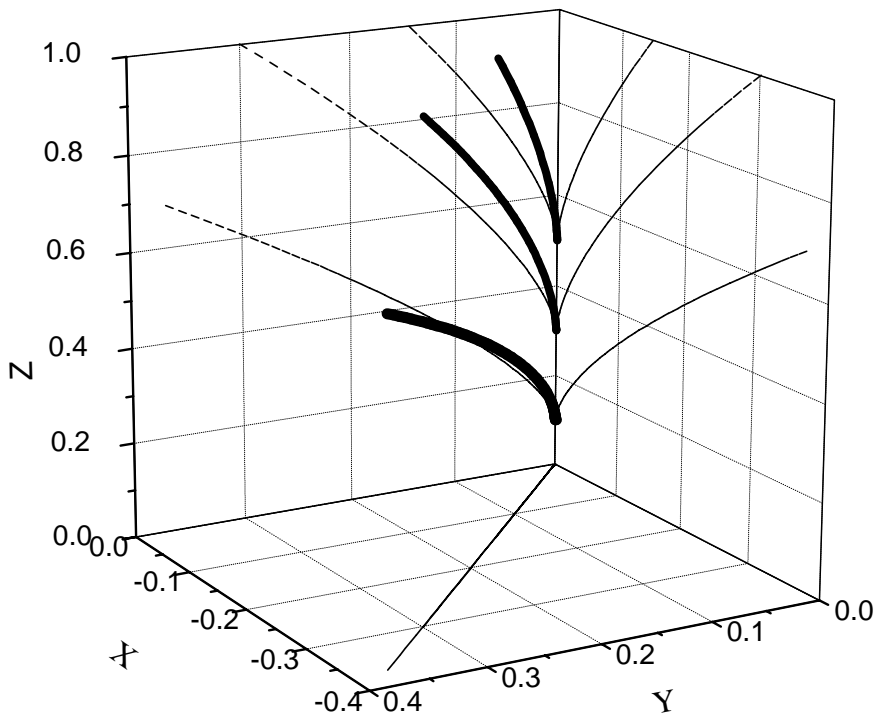

Fig. 4. Exponential trajectories of particles originating at 3 different initial positions for the case in which $\mathcal{D}=0$ and $\Omega_{x}=\Omega_{y}$.

and

$z(t)=2 A_{z} \mathrm{e}^{a t}+C_{z} \mathrm{e}^{-2 a t}$.

Regardless of the value of $a$, solutions for this case will grow exponentially.

\subsubsection{Mixed solutions}

Particles will move on exponentially growing or decaying oscillatory trajectories when both $\mathrm{a}$ and $\mathrm{b}$ are real and non-zero. This will be the case with $\mathcal{D}>0$, if the magnetic field has at least components in the $y$ and $z$ directions.

Under these conditions, and for all values of $K$ and field configurations the $s_{1}>0$ and $s_{2}<0$. This implies that $a$ and $b$ are positive. Thus, in solutions (21)-(23) the purely exponential term of the form $C_{w} \mathrm{e}^{-2 a t}$ for $w=$ $x, y$, or $z$ only contributes initially and rapidly becomes negligible in comparison to the oscillatory terms.

Examples of this behavior are seen in Figs. 5 and 6 . The first case corresponds to the simplest geometry for this type of solution, that is a magnetic field with $\Omega_{x}=0$ and $\Omega_{y}=\Omega_{z}$. The second case, Fig. 6 , corresponds to particle trajectories in a magnetic field with all three components being equal. The velocity shear is assumed to be $K=0.1 \Omega$ in both cases but the precise choice will be discussed later.

Let us analyze solutions for the simplest configuration although more general cases are qualitatively similar. When $\Omega_{x}=0$, calculating $s_{1}$ and $s_{2}$ from (17) and (18) we obtain $a \approx 0.03 \Omega$ and $b \approx 0.6 \Omega$ The particle trajectories will be described by Eqs. (21)-(23) with all coefficients real. Close analysis of the solutions indicates that these can be written as

$x(t)=\left(2 A_{x} \cos b t-2 B_{x} \sin b t\right) \mathrm{e}^{a t}+x_{\mathrm{c}}(t)$,

$y(t)=\left(2 A_{y} \cos b t-2 B_{y} \sin b t\right) \mathrm{e}^{a t}+y_{\mathrm{c}}(t)$,

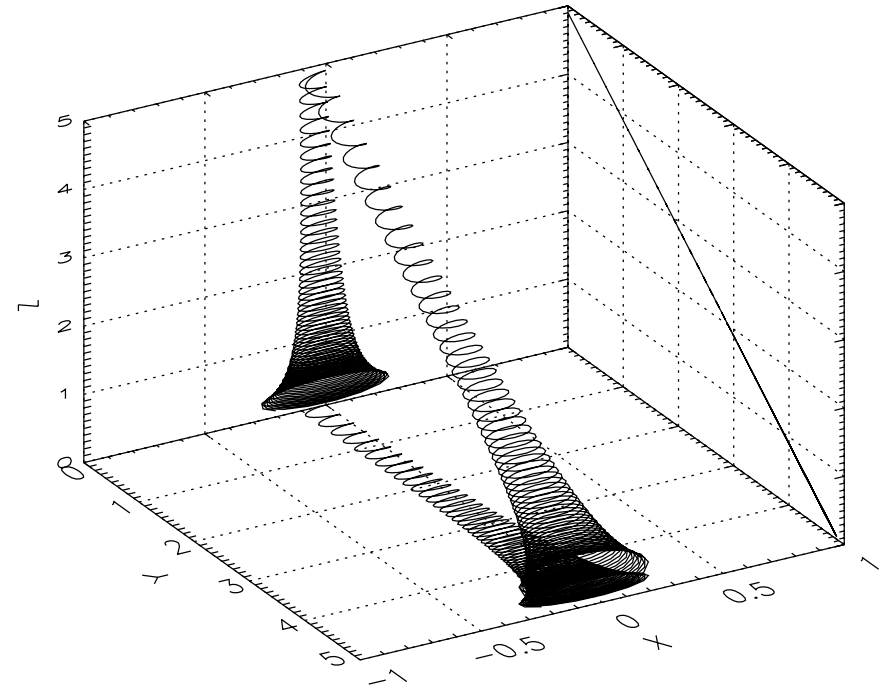

Fig. 5. Trajectory of mixed type, exponential and oscillatory, for a particle originating at the top of the boundary layer for the case in which $\mathcal{D}>0$ and $\Omega_{x}=0, \Omega_{y}=\Omega_{z}$.

and

$z(t)=\left(2 A_{z} \cos b t-2 B_{z} \sin b t\right) \mathrm{e}^{a t}+z_{\mathrm{c}}(t)$,

where the position of the center of oscilation is given by

$x_{\mathrm{c}}(t)=C_{x}\left(\mathrm{e}^{-2 a t}-1\right)-2 A_{x}$,

$y_{\mathrm{c}}(t)=C_{y}\left(\mathrm{e}^{-2 a t}-1\right)-2 A_{y}$,

and

$z_{\mathrm{c}}(t)=C_{z} \mathrm{e}^{-2 a t}$

The particle will execute oscillatory motion with an amplitude that grows exponentially at a rate a while the center of the oscillation moves according to Eqs. (42)-(43). Note that since $a>0$, as time progresses, the center of the oscillation will lead to a finite, non-zero value in $x$ and $y$ depending on the initial position and the field configuration. Also, within the restrictions of the case analyzed in this subsection particles will move toward $z=0$ regardless of the magnetic field configuration or the velocity shear.

We will return to the discussion of the implications of these redistribution and acceleration of the particles in the next section.

\subsection{Results for $\mathcal{D}<0$}

When the discriminant $\mathcal{D}$ is negative then, according to Eqs. (17) and (18), $s_{1}$ and $s_{2}$ will be the conjugate of each other. This implies that $a$ is real and $b$ is imaginary and thus all roots of Eq. (10) are real and the solutions are purely exponential.

Once again we will analyze in detail a simple case for this regime when $\Omega_{y}=\Omega_{z}=0$. For such configuration, the condition $\epsilon=K / \Omega>1$ is equivalent to a negative 


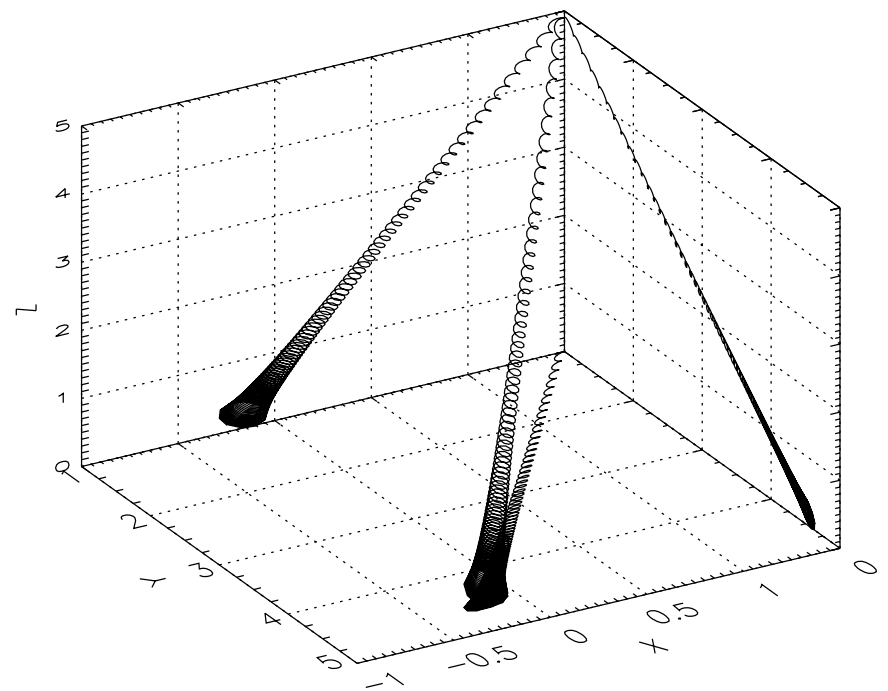

Fig. 6. Trajectory of mixed type for a particle originating at the top of the boundary layer for the case in which $\mathcal{D}>0$ and $\Omega_{x}=\Omega_{y}=\Omega_{z}$.

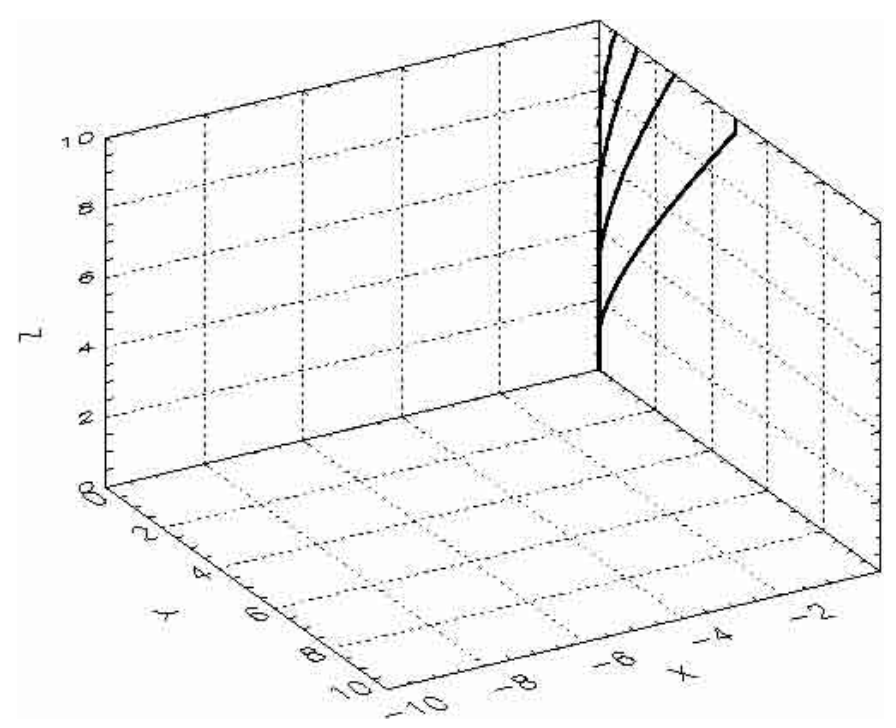

Fig. 7. Trajectories of particles originating at 4 different initial positions for the case in which $\epsilon>1$ and $\Omega_{y}=\Omega_{z}=0$.

discriminant. Solutions, shown in Fig. 7 can be written as:

$x(t)=0$,

$y(t)=\frac{\epsilon z_{\mathrm{o}}}{2(\epsilon-1)^{3 / 2}}\left(\mathrm{e}^{\varpi t}-\mathrm{e}^{-\varpi t}\right)-\frac{K z_{\mathrm{o}} t}{2(\epsilon-1)}$,

and

$z(t)=\frac{\epsilon z_{\mathrm{o}}}{2(\epsilon-1)}\left(\mathrm{e}^{\varpi t}+\mathrm{e}^{-\varpi t}\right)-\frac{z_{\mathrm{o}}}{\epsilon-1}$,

where $\varpi=\Omega(\epsilon-1)^{1 / 2}$. After a few timescales $\sim 1 / \varpi$ the solution is dominated by the exponential growth term with the particle escaping rapidly from the source region.

More general magnetic field orientations, with $\mathcal{D}<0$ lead to similar solutions. An example corresponding to $\Omega_{x}=\Omega_{y}=\Omega_{z}$ and $K=1.6 \Omega_{x}$ is shown in Fig. 8 .

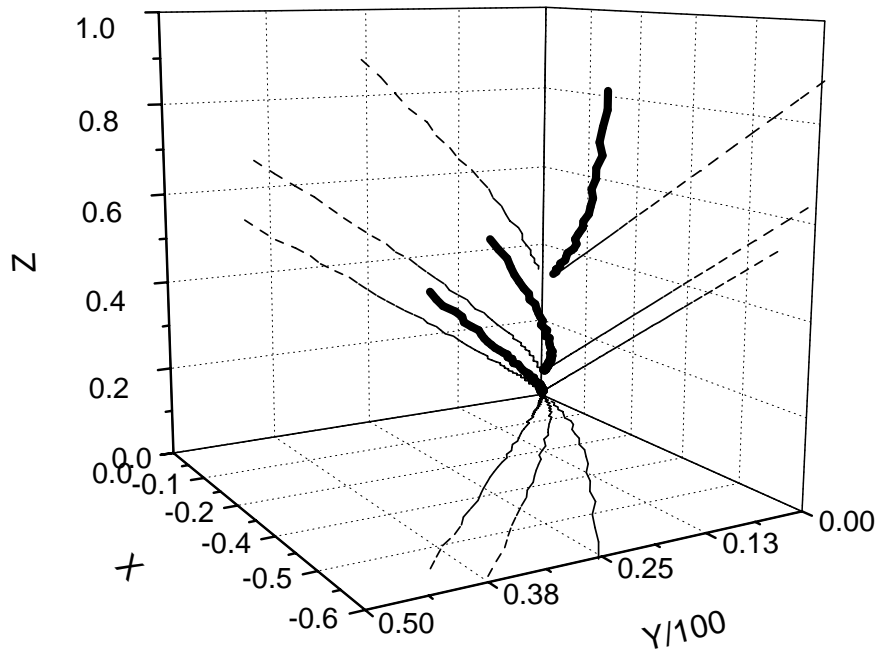

Fig. 8. Trajectories of particles originating at 3 different initial positions for the case in which $\epsilon>\epsilon_{\text {crit }}$ and $\Omega_{x}=\Omega_{y}=\Omega_{z}$.

\section{Discussion}

The most notable results that have been described are related to the exponential acceleration and the migration of the contaminant ions that move within the velocity shear. Acceleration on mixed trajectories, with a constant velocity drift, will only occur whenever the magnetic field has non-zero $B_{y}$ and $B_{z}$ components. If either of these components is zero, exponential particle acceleration can take place if $\epsilon>1$.

In this section we discuss the conditions and limits of the mechanism of particle acceleration.

\subsection{Conditions for particle acceleration}

As we have shown above, particle acceleration can take place whenever $\Omega_{y}, \Omega_{z}$ and $K$ are different from zero. For a given flow and magnetic field configuration the rate at which particles are accelerated depends on their mass. For example, for the particular case shown in Fig. 5 the amplitude of the oscillations, both in position and velocity, grows at a rate $a \approx 0.03 q \mathrm{~B} / \mathrm{mc}$ and hence, for this configuration, the more massive ions will be accelerated at a lesser rate.

For cases in which there are purely exponential trajectories, such as those shown in Figs. 4 and 7, there is a critical value of $\epsilon$,

$\epsilon_{\text {crit }}=1+27\left[\frac{K \Omega_{y} \Omega_{z}}{2 \Omega^{3}}\right]^{2 / 3}$,

at and above which, such solutions are obtained.

For a given magnetic field and flow properties, ions with mass such that $\epsilon$ is greater than the critical value, will move on exponential trajectories instead of the oscillatory motion derived from previous studies.

For the simple magnetic configuration when the field is in the $x y$ plane the condition for particle acceleration is 
simply $\epsilon>1$. In terms of the ion mass the condition for having exponential trajectories is:

$\frac{m_{\mathrm{p}} c K B_{x}}{q\left(B_{x}^{2}+B_{y}^{2}\right)}>1$

So the critical mass for this particular magnetic configuration is

$m_{\text {crit }}=\frac{q\left(B_{x}^{2}+B_{y}^{2}\right)}{c K B_{x}}$.

Ions with mass less than this critical value will execute cycloidal motion drifting with the flow, while ions with mass greater than the critical value will describe orbits of exponentially increasing size and velocity.

\subsection{Limits of particle acceleration}

Accelerated ions also migrate in the $z$ direction, upwards for purely exponential trajectories, or downwards for oscillatory exponential trajectories as those described in Sect. 3.1.3. The limits for particle acceleration are then set by the fact that ions will eventually leave the region where the velocity shear is present.

The width of the velocity shear region in our model can be prescribed or determined by the adopted velocity gradient, $K$ and an assumed velocity for the free flowing wind, i.e. for the wind outside the velocity shear, $U_{\mathrm{w}}$. We adopt the latter approach so that the width of the shear region is given by $H=U_{\mathrm{w}} / K$.

Our analysis, and the predicted acceleration, will no longer be applicable when an ion reaches the bottom or the top of the shear layer. Ions moving on exponential trajectories will be accelerated when they move within the width $H$. Ions on mixed trajectories will undergo exponentially growing oscillations moving downwards eventually reaching the bottom of the velocity shear, i.e. $z=0$.

We analyze the maximum acceleration possible for both cases separately by looking at the behavior of the particle kinetic energy as the particles migrates in the $z$ direction. Exponential trajectories are exemplified by the case in which the magnetic field is: $\boldsymbol{B}=(B, 0,0)$ and $\epsilon>1$. Acceleration for particles on mixed type trajectories is exemplified by the special case in which $B_{x}=0$ and $B_{y}=B_{z}$.

\subsection{1. $\boldsymbol{B}=(B, 0,0)$}

The trajectory of particles subject to this configuration is obtained from Eqs. (32)-(34) by taking $\Omega_{z}=0$. Rewriting in terms of $\epsilon$ the particle velocity as a function of time is:

$v_{y}(t)=\frac{K z_{\mathrm{o}}}{1-\epsilon}(1-\cos \omega t)$

and,

$v_{z}(t)=\frac{K z_{\mathrm{o}}}{\sqrt{1-\epsilon}} \sin \omega t$

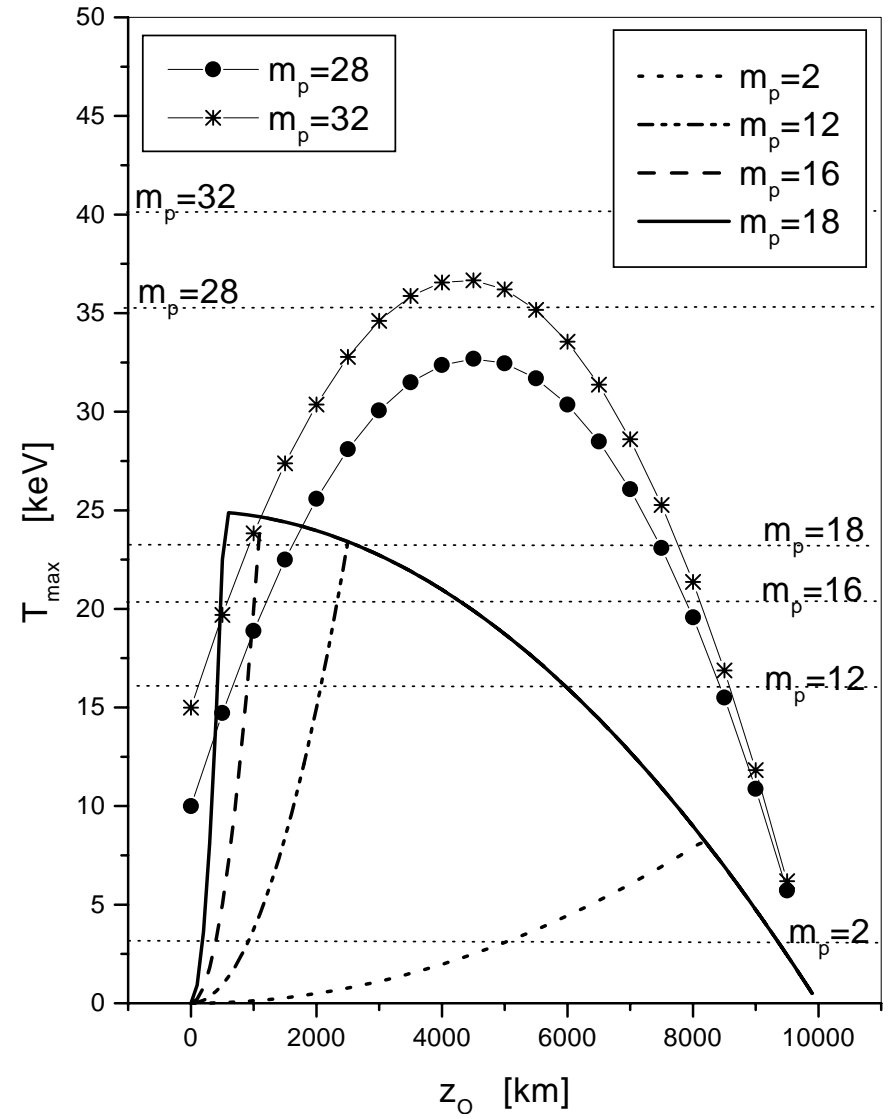

Fig. 9. Maximum kinetic energy attained by the ions as function of the initial position in the gradient. Each curve corresponds to different ion mass. The horizontal lines are the maximum energy attained by the ions in a flux without shear. In this case we considered $B=10 \gamma, H=10^{4} \mathrm{~km}, U_{\mathrm{w}}=500 \mathrm{~km} \mathrm{~s}^{-1}$.

with no component in the $x$ direction for this particular case. The kinetic energy for exponential solutions $(\epsilon>1)$ as a function of time is given by

$T=\frac{m K^{2} z_{\mathrm{o}}^{2}}{2(1-\epsilon)}\left[2-2 \cosh \varpi t-\epsilon \sinh ^{2} \varpi t\right]$.

The maximum kinetic energy attained by ions on exponential trajectories is obtained after finding the time at which they reach the top of shear layer. The calculation of the time to reach $H$, and the resulting kinetic energy, are easily carried out but it does not lead to a simple analytical expression. The maximum kinetic energy is a function of the mass of the accelerated ions as well as the magnetic field strength (through $\varpi$ ), the velocity gradient $K$, the streaming wind velocity, which determines $H$, and the position $z_{0}$ where the ion is born. The maximum kinetic energy is shown in Fig. 9 as a function of the ion mass and the initial position for the case relevant to the solar wind flow past a non-magnetic planet.

Figure 9 shows the maximum energy attained by a particle as function of initial position, $z_{0}$, and ion mass. In this figure we can observe two types of behaviour for the maximum kinetic energy depending on the value of epsilon or $m_{\mathrm{p}}$, since $\epsilon=K m_{\mathrm{p}} c / q B$ in this configuration. 
For the case of exponential acceleration, i.e. if $m_{\mathrm{p}}$ is such that $\epsilon>1$, the maximum kinetic energy of the particles is always less than the kinetic energy of ions in the free streaming wind. Alltough the acceleration increases with $z_{0}$, ions born above a certain height escape from the boundary layer so quickly that no significant acceleration is achieved. Hence, particles on purelly exponential trajectories are not even accelerated to the velocity of the freely streaming wind.

It is to be noted that a greater maximum kinetic energy can be attained by particles with mass such that $\epsilon<1$, which move on cycloidal trajectories. This is also shown in Fig. 9 where it is seen that $T_{\max }$ increases as $z_{0}$ increases until, again, particles exit the boundary layer before they attain the maximum velocity possible in their gyromotion.

The behavior of the kinetic energy for diferent magnetic fields and velocity gradients leading to exponential trajectories, is qualitatively similar.

\subsection{2. $\boldsymbol{B}=\left(0, B_{y}, B_{z}\right)$}

As discussed in Sect. 3.1.3 particle acceleration will also occur when both $B_{y}$ and $B_{z}$ are different from zero. Ions will then move on exponentially growing oscillations given by mixed type solutions. The particles will migrate downwards until they reach the bottom of the boundary layer which will set the limit of the acceleration process.

Once again we illustrate our results looking at the simplest configuration, namely, that in which $\boldsymbol{B}$ is entirely in the $y z$ plane and the components have equal values. The particle trajectories in this case are given by Eqs. (39)(44). The calculation of the kinetic energy is a direct but rather tedious exercise leading to a complicated expression. The evolution of the particle kinetic energy is exemplified by the case of ion mass $m_{\mathrm{p}}=28 m_{\mathrm{H}}$, magnetic configuration, $B_{z}=B_{y}$, and velocity gradient, $K=0.001 \Omega$, shown in Fig. 10. The particles gain kinetic energy as they migrate downwards until they reach the bottom of the acceleration region.

The maximum kinetic energy attained by particles in this configuration can be much greater than that of particles in the cases discussed in the previous section. This is due to the fact that particles, specially those starting near the upper bound of the boundary layer, spend a great amount of time in the region, gaining energy in each oscillation.

The maximum kinetic energy depends strongly on the ion mass, the initial position, and the field configuration. Figure 11 shows the maximum kinetic energy attained by a particle starting from $z=H$ as a function of its mass and the magnetic field direction is in the $y z$ plane, characterized by the angle $\phi=\operatorname{atan}\left(B_{y} / B_{z}\right)$. In Fig. 11 we can see that the maximun kinetic energy attained by the ion increases with $\phi$ angle; as $\phi$ increases, the drift in the $z$ direction decreases and the ion stays a longer time in the aceleration zone, e.g., if $\phi=70^{\circ}$ and the ion mass is $m_{\mathrm{p}}=28 m_{\mathrm{H}}$, the ion arives to the bottom of the region

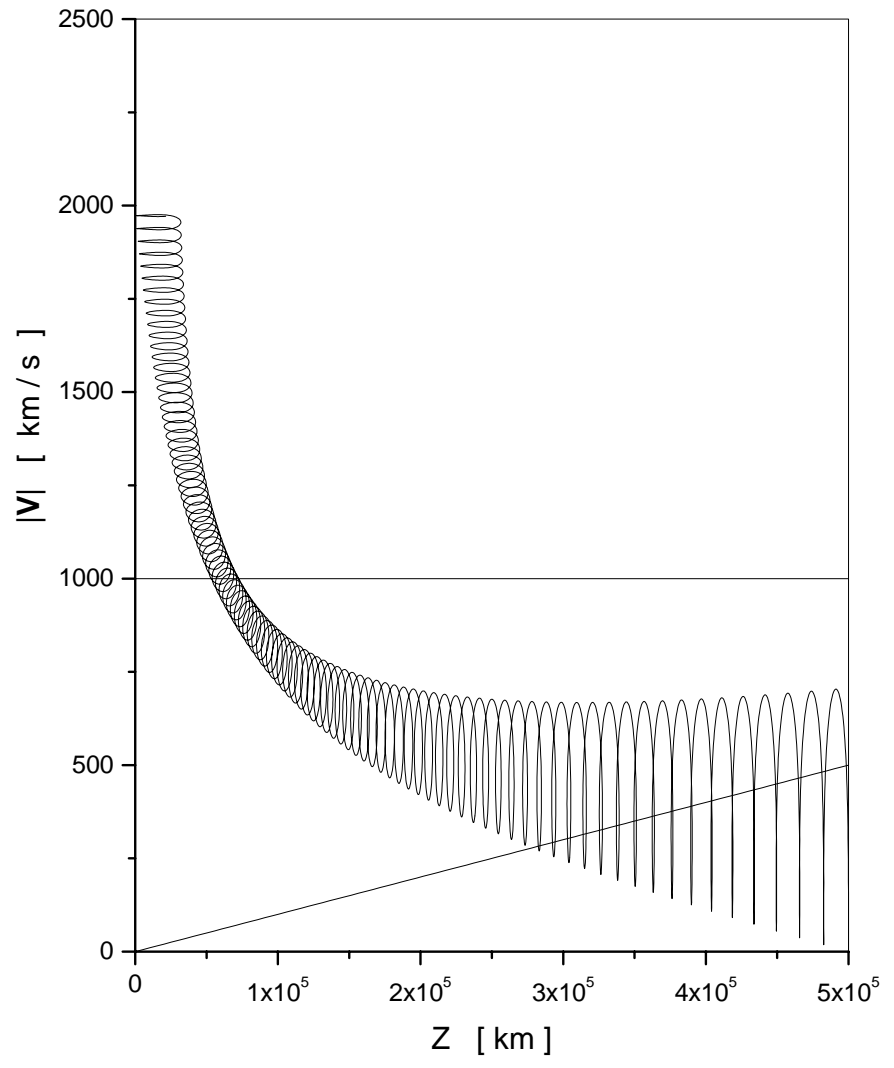

Fig. 10. An example of the evolution of the total speed of a particle that "born" in the highest layers of a velocity shear descending to layers of low velocity. In this case $B=10 \gamma$, $H=5 \times 10^{5} \mathrm{~km} . U_{\mathrm{w}}=500 \mathrm{~km} \mathrm{~s}^{-1}$. The magnetic configuration is $B_{z}=B_{y}, B_{x}=0$. The horizontal line is the speed of ions in the free streaming wind. The inclined solid line is the local background flow velocity.

with an energy 25 times greater the energy of similar ions in the free streaming wind. Furthermore, the energy of the ion is approximately 3000 times the energy of particles in the background flow at a height of $50000 \mathrm{~km}$.

\section{Applications}

Evidence of velocity shears in the region of interaction of the solar wind with plasma obstacles is available from measurements made at Venus, Mars and comets Giacobinni-Zinner, Halley and Gregg-Skjellerup. As a whole the velocity shear is seen along the flanks and downstream from the plasma obstacle with a thickness that increases with distance along the tail direction. In the near wake of Venus and Mars the velocity shear has a 1000-2000 km width (Bridge et al. 1967; Romanov et al. 1979; Lundin et al. 1991) and thus is much smaller than the ( 100000 km values that have been inferred from measurements made in cometary plasma environments (Johnstone et al. 1986; Balsiger et al. 1986; Gloecker et al. 1986). An important feature of the velocity shear seen in all these cases is that it is bounded by a sharp plasma transition across which there is a strong change in the velocity gradient in the direction transverse to the solar wind flow 


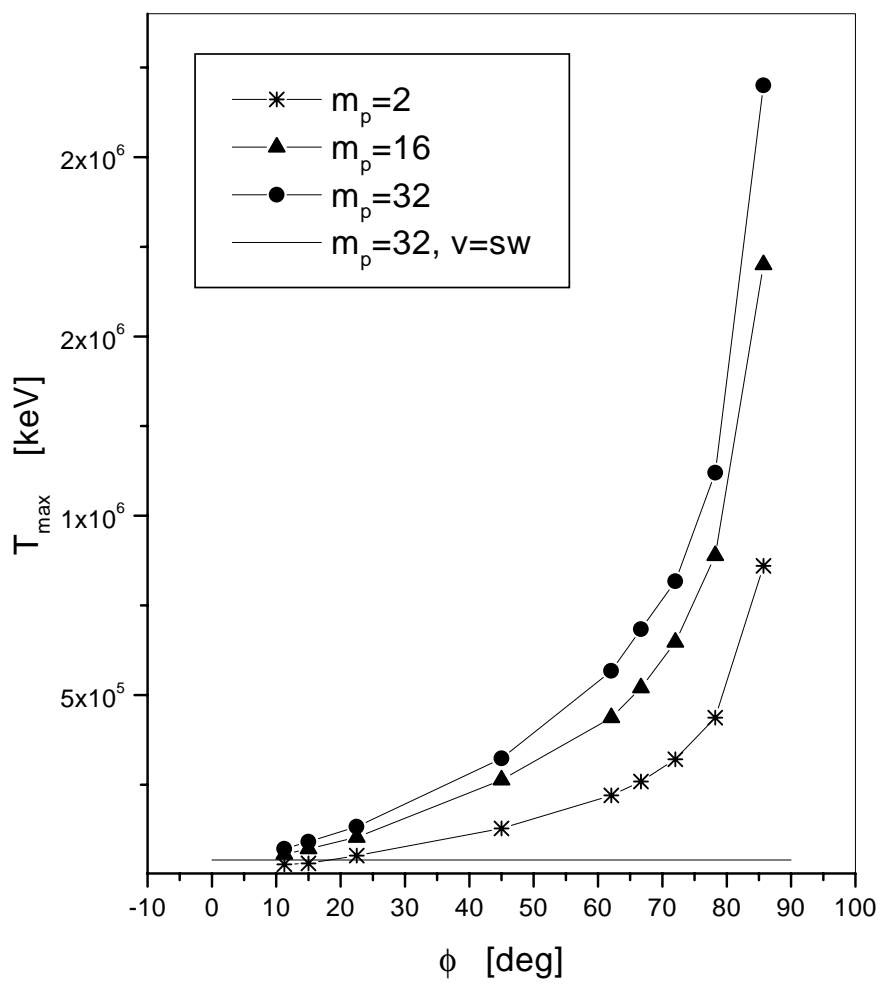

Fig. 11. Maximum kinetic energy attained by the ions as function of the angle $\phi(\phi=\operatorname{atan}(B y / B z))$. Each curve corresponds to different ion mass. In this case we considered $B=10 \gamma, H=5 \times 10^{5} \mathrm{~km}$ and $U_{\mathrm{w}}=500 \mathrm{~km} \mathrm{~s}^{-1}$.

(Pérez-de-Tejada et al. 1995). Measurements show that the flow speed begins a strong decrease from conventional $300-400 \mathrm{~km} \mathrm{~s}^{-1}$ solar wind values at that boundary to a small fraction seen closer to the plasma obstacle. Further information is that across the velocity shear the plasma is hotter and becomes more turbulent than what it is seen above it. At the same time there are indications that the plasma flux intensity within the velocity shear becomes much smaller than the values measured further outside.

While these variations in the plasma properties of the solar wind together with the transterminator flow seen in the upper ionosphere (Knudsen et al. 1980) are consistent with the overall behavior expected from the effects of momentum transport through viscous-like processes (Pérez-de-Tejada 1986), there are unrelated observations that reveal further important conditions within the shear layer. In particular, measurements conducted in the Mars magnetosheath with a particle detector system of the Phobos spacecraft indicate the presence of energetic particle fluxes at the lowest $(\sim 900 \mathrm{~km})$ altitudes from Mars that were approached along trajectories near the flank regions. These include enhanced values in the $30-200 \mathrm{keV}$ energy range far above those expected from the standard pick-up process for $\mathrm{O}+$ ions in the freestream solar wind (Afonin et al. 1989). While it has been suggested that such accelerated particles may be related to a pronounced compression of the interplanetary magnetic field lines close to the planet it is important to point out that the location where they are observed agrees very closely with the region where the flow speed decreases to very small values. Measurements conducted with the ASPERA experiment in the Phobos spacecraft show, for example, that along that section of the Phobos trajectory the speed of the shocked solar wind decreases to very low $\left(\sim 50 \mathrm{~km} \mathrm{~s}^{-1}\right)$ values (Lundin et al. 1991). The presence of a sharp velocity shear in that region of space may thus provide conditions suitable to the local acceleration of plasma particles as was discussed above. A similar agreement can also be inferred from measurements conducted in cometary plasma environments. Energetic particle fluxes have been observed within the wide velocity shear across the plasma tail of comets Giacobinni Zinner and Halley (Gloecker et al. 1986). Thus it is conceivable that particle acceleration within a velocity shear may be an important contributor to the population of energetic particles seen in those regions of space.

\section{Summary}

We have analyzed the trajectories of pick-up ions in linear velocity shears as those expected as the solar wind flows around nonmagnetic bodies (Venus, Mars, comets). The most interesting result we found, exclusively present in velocity shears, is the possibility of accelerating pick-up ions to velocities much greater than those in the free streaming wind. We have discussed the conditions and limits of the acceleration mechanism and speculated on possible applications to explain the presence of high energy ions in various scenarios.

Acknowledgements. We thank CONACYT-México for support for E.P. through a scholarship for graduate studies. M.R. acknowledges support from CONACYT grant J32990E.

\section{References}

Afonin, V., et al. 1989, Nature, 341, 616

Balsiger, H., et al. 1986, Nature, 321, 330

Bame, S., et al. 1986, Science, 232, 356

Bridge, H., et al. 1967, Science, 158, 1669

Gloecker, G., et al. 1986, Geophys. Res. Lett., 13, 251

Johnstone, A., et al. 1986, Nature, 321, 344

Knudsen, W., et al. 1980, J. Geophys. Res., 85, 7803

Landau, L. D., \& Lifshitz, E. M. 1987, Fluid Mechanics (Pergamonn press, Oxford)

Lundin, R., et al. 1991, Geophys. Res. Lett., 18, 1059

Parker, E. 1958, Phys. Fluids, 1, 171

Pérez-de-Tejada, H. 1986, J. Geophys. Res., 91, 8039

Pérez-de-Tejada, H., et al. 1995, J. Geophys. Res., 100, 14523

Pérez-de-Tejada, H., \& Durand-Manterola, H. 1996, Phys. Plasmas, 3(2), 639

Pérez-Tijerina, E., et al. 2000, A\&A, 354, 321

Perez-Tijerina, E. 1999, B.Sc. Thesis, Universidad Autóma de Baja California, México

Romanov, S., et al. 1979, Cosmic Res., 16, 603

Shapiro, V., et al. 1989, J. Geophys. Res., 100, 21 